\title{
Recovery of right ventricular function and strain
}

\author{
Johan H. C. Reiber ${ }^{1}$ \\ Published online: 2 March 2022 \\ (c) The Author(s), under exclusive licence to Springer Nature B.V. 2022
}

Dear reader,

For this March 2022 issue, I would like to recommend the paper by A. Van Veelen and co-authors from a number of university medical centers under the supervision of A Hirsch from the Erasmus University Medical Center in Rotterdam, The Netherlands [1]. The topic of this paper is the investigation of the right ventricular (RV) function in STEMI patients with concurrent chronic total occlusion (CTO). They analyzed a total of 174 patients as a substudy of the EXPLORE trial, with serial magnetic resonance imaging of the right ventricle (at baseline and 4 months follow-up) and subdivided the total patient population in 3 subgroups depending on the presence of the CTO and culprit lesion in the RCA or no-RCA. They measured the Tricuspid annular plane systolic excursion (TAPSE), RV ejection fraction (RVEF), RV global longitudinal strain (GLS) and free wall longitudinal strain (FWLS). An example of their measurements is presented in Fig. 1, which is Fig. 2 from their manuscript.

The main conclusion from their research is that the RV parameters significantly improved over time in patients with acute or chronic RCA occlusion, but not in the no-RCA patients. Furthermore, the RV free wall longitudinal strain (FWLS) was the only RV parameter able to discriminate between acute ischemic and non-ischemic myocardium. For further details, I refer of course to the manuscript.

I wish you much pleasure in reading this and the other papers in this issue of the International Journal of Cardiovascular Imaging.
Johan H. C. Reiber

J.H.C.Reiber@lumc.nl

1 Leiden University Medical Center, Leiden, The Netherlands 


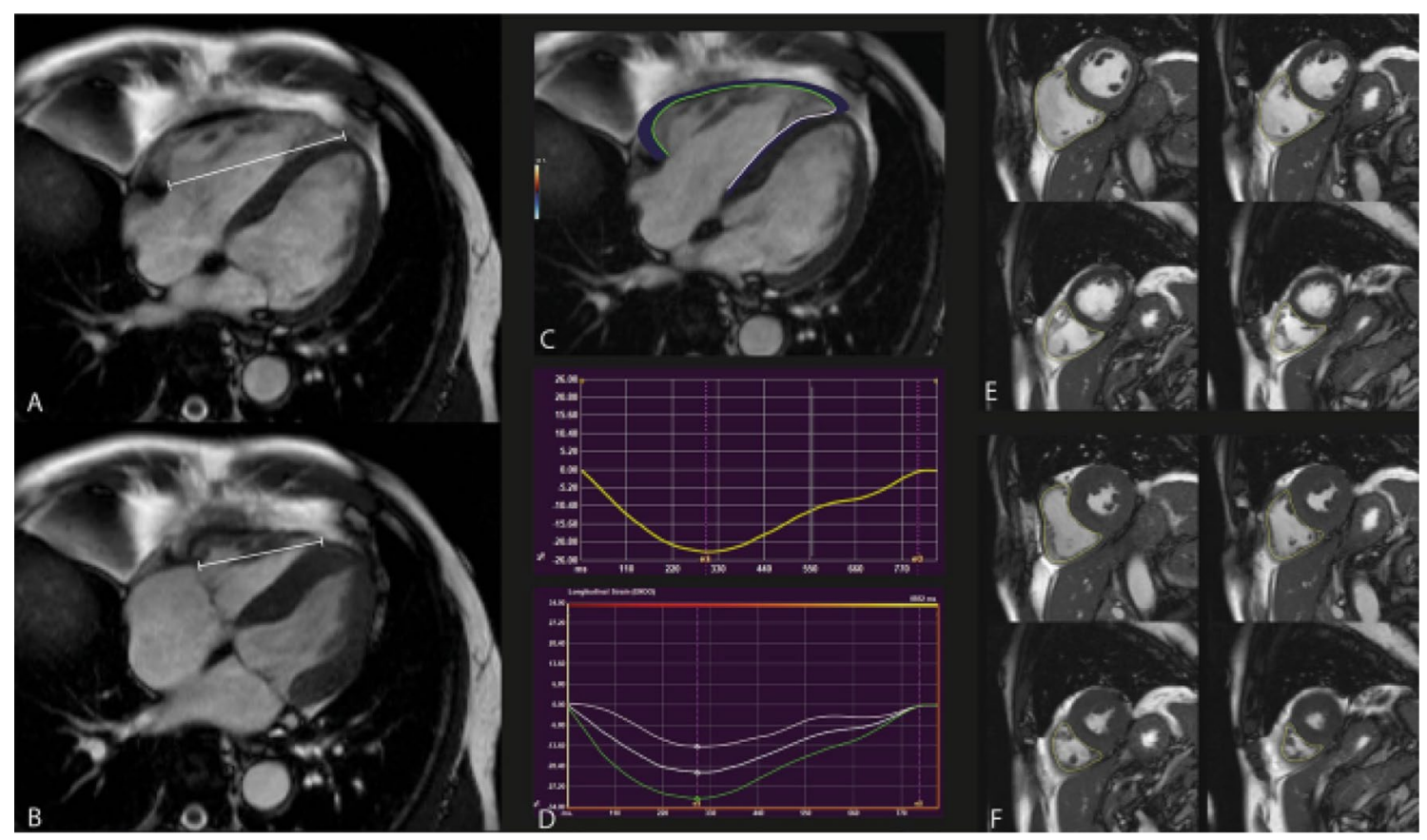

Fig. 1 Measurement of right ventricular function parameters. A and B End-diastolic length (A) and end-systolic length (B) measurement for the calculation of the tricuspid annular plane systolic excursion; C Right ventricular strain measurement using feature tracking, with the green line indicating tracking of the free wall and white line indicating the septum tracking; D Strain curves, with the upper curve

\section{Reference}

1. van Veelen A, Elias J, van Dongen IM et al (2021) Recovery of right ventricular function and strain in patients with ST-segment elevation myocardial infarction and concurrent chronic total occlusion. Int J Cardiovasc Imaging. https://doi.org/10.1007/ s10554-021-02423-9 indicating the general RV strain curve and the lower curve indicating the RV strain curves divided into septum (white), average (white) and free wall (green). eS indicates end-systolic and $\mathrm{eD}$ indicates end-diastolic; E, F Right ventricular contours in the short-axis end-diastole (E) and end-systole (F) for RV volume measurements

Publisher's Note Springer Nature remains neutral with regard to jurisdictional claims in published maps and institutional affiliations. 